\section{Cureus}

Received 01/20/2019

Review began 02/08/2019 Review ended 02/10/2019 Published 02/13/2019

\section{(c) Copyright 2019}

Farag et al. This is an open access article distributed under the terms of the Creative Commons Attribution License CC-BY 3.0., which permits unrestricted use, distribution, and reproduction in any medium, provided the original author and source are credited.

\title{
Tetrasomy 8 Associated with a Poor Prognosis in Acute Monoblastic Leukemia: A Case Report
}

\author{
Fady Farag ${ }^{1}$, Rewais Morcus ${ }^{1}$, Preethi Ramachandran ${ }^{2}$, Karan Josan ${ }^{2}$, Jen Chin Wang ${ }^{2}$ \\ 1. Internal Medicine, Brookdale University Hospital and Medical Center, Brooklyn, USA 2. Oncology, \\ Brookdale University Hospital and Medical Center, Brooklyn, USA
}

$\square$ Corresponding author: Rewais Morcus,rmorcus@bhmcny.org

Disclosures can be found in Additional Information at the end of the article

\section{Abstract}

We report a case of a 47-year-old male from West Africa who presented with sepsis and was found to have acute monoblastic leukemia associated with tetrasomy 8 detected on bone marrow samples. This was the only chromosomal abnormality found. Tetrasomy 8 is a rare genetic finding that has been reported in acute myeloid leukemia (AML), predominantly the monocytic lineage. It carries a poor prognosis with a high mortality rate.

Categories: Genetics, Oncology

Keywords: acute myeloid leukemia, tetrasomy 8, poor prognosis, karyotyping, aml

\section{Introduction}

Trisomy 8 is one of the most encountered chromosomal aberrations found in acute myeloid leukemia (AML) and myelodysplastic syndrome [1]. However, tetrasomy 8 is a rare genetic finding with only 33 cases reported [2]. Tetrasomy 8 was linked to the monocytic lineage and was found to be the single genetic abnormality in about half the reported cases. It was found to carry poor prognosis with an aggressive course [3]. We report here a case of AML associated with tetrasomy 8 in a patient who presented with sepsis secondary to community-acquired pneumonia and malaria.

\section{Case Presentation}

We report a case of a 47-year-old male patient from Mali, West Africa, who presented with intermittent fever, chills, increased fatigue, decreased appetite, and diffuse back and abdominal pain for three weeks. He reported a 35-pound weight loss over three months. He had a history of malarial infection six months before, which was treated. He endorsed traveling through Africa until a recent illness. At the time of admission, his temperature was $102.3 \mathrm{~F}$, blood pressure was 103-110/59-64 mmHg, and heart rate was $92-113 \mathrm{bpm}$, saturating at $99 \%$ on room air. A blood examination showed a hemoglobin level of 4.4, a mean corpuscular volume (MCV) of 87.5, a platelet count of 13, a white blood cells (WBC) count of 6.4, and an international normalized ratio (INR) of 1.73. Other values included serum sodium 132, lactate 4.6, alkaline phosphatase 133, total bilirubin 1.9, direct bilirubin 1.6, and albumin 2.8. The malarial screen was negative. A chest X-ray showed bilateral lower lobes infiltrates consistent with pneumonia. A computed tomography (CT) scan of the chest, abdomen, and pelvis showed mild bilateral areas of focal infiltrates and consolidation consistent with pneumonia and small right scrotal hydrocele. A peripheral blood smear (Figure 1) showed few monoblasts, few dysplastic features, and erythrocytes with intracellular inclusions. He received supportive care with blood and 


\section{Cureus}

platelet transfusions. He was treated with intravenous ceftriaxone and intravenous azithromycin for community-acquired pneumonia and atovaquone for malaria.
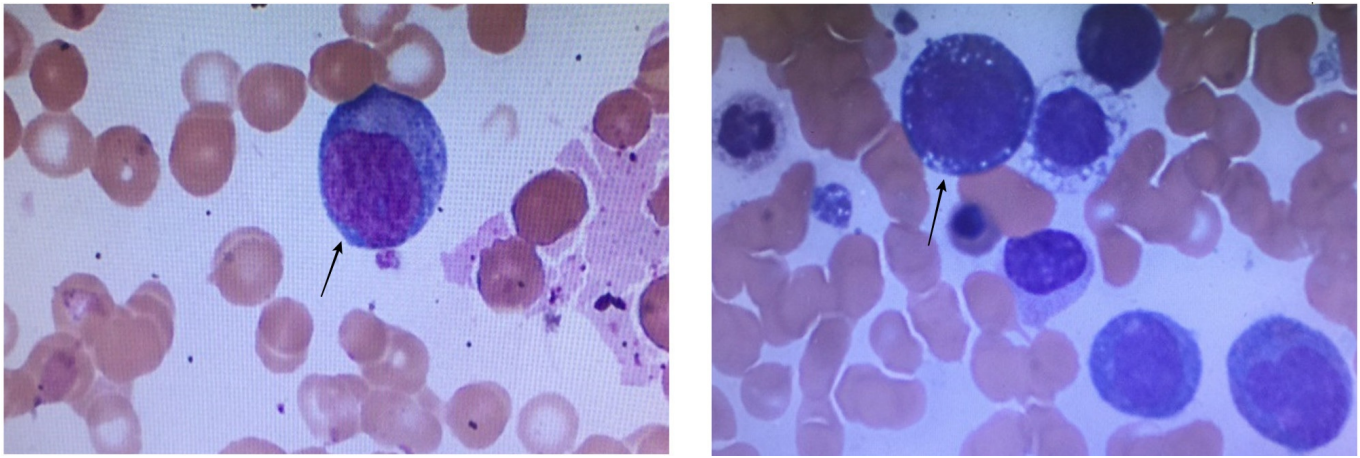

\section{FIGURE 1: Peripheral Blood Smear Picture}

A bone marrow aspirate was done (Figure 2), and the smear showed hypercellular marrow with monoblasts, erythroblasts, maturation defect, and few megakaryocytes. Further examination showed hypercellular marrow for age with sheets of immature mononuclear cells (blasts and monocytes) diffusely occupying marrow cavities. Table 1 shows the differential count of the bone marrow cells. Blasts comprised approximately 50\% of marrow elements, confirmed by immunostaining with CD34 and CD117. Monocytes comprised approximately $20 \%-30 \%$ of marrow elements. The blasts were medium to large with increased nucleus-to-cytoplasm (N/C) ratio, fine chromatin, and prominent nucleoli.
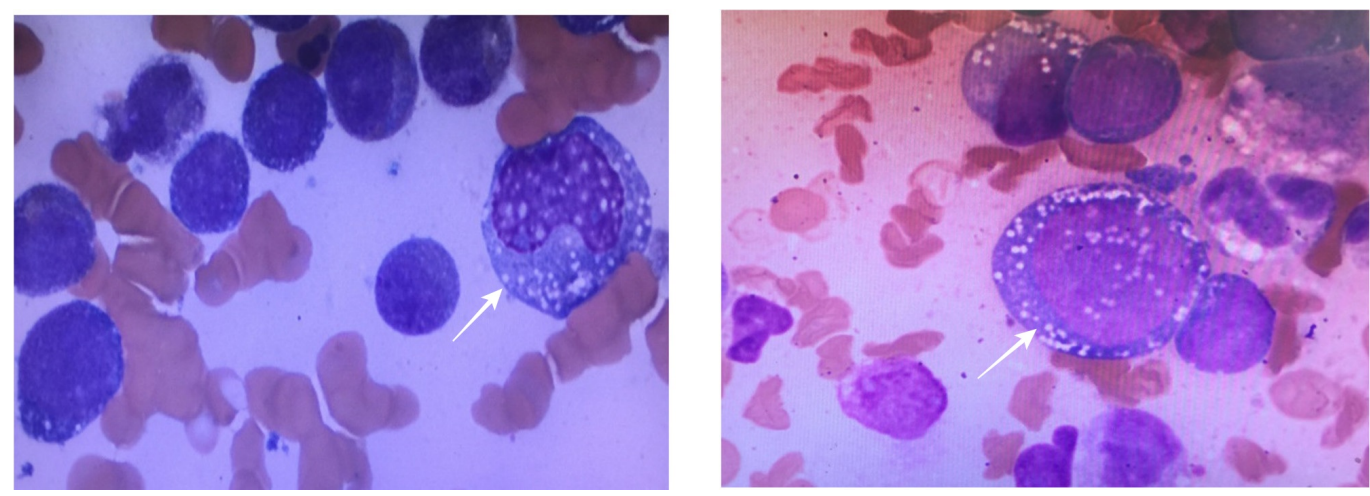

\section{FIGURE 2: Bone Marrow Aspiration Showing Monoblasts}

Monoblasts are the largest blasts of all the hematopoeitic cell lines present in the bone marrow. They have a large, round, centrally-placed nucleus with soft, fine-stranded chromatin. They normally have a single, large, prominent nucleolus. The cytoplasm is very generous and has a fine, grainy texture.

Table 1 presents the bone marrow differential count. 


\section{Cureus}

\begin{tabular}{|c|c|c|}
\hline Cell type & Results & Normal range \\
\hline Myeloblasts & $17 \%$ & $0-3 \%$ \\
\hline Promyelocytes & $5 \%$ & $2-8 \%$ \\
\hline Myelocytes & $9 \%$ & $10-13 \%$ \\
\hline Metamyelocytes & $8 \%$ & $10-15 \%$ \\
\hline Neutrophils / Bands & $15 \%$ & $25-40 \%$ \\
\hline Monocytes & $22 \%$ & $0-1 \%$ \\
\hline Eosinophils & $4 \%$ & $1-3 \%$ \\
\hline Basophils & $1 \%$ & $0-1 \%$ \\
\hline Lymphocytes & $8 \%$ & $10-15 \%$ \\
\hline Plasma Cells & $1 \%$ & $0-1 \%$ \\
\hline Pronormoblasts & $1 \%$ & $0-2 \%$ \\
\hline Normoblasts & $9 \%$ & $15-25 \%$ \\
\hline
\end{tabular}

\section{TABLE 1: Bone Marrow Differential Count}

These results were consistent with a diagnosis of acute myeloid leukemia (non-APL), best classified as acute myelomonocyte leukemia (AMML). Flow cytometry analysis from the aspirate showed blasts (12\%-15\%) that were positive for CD34 (partial), CD117, HLA-DR, CD13, CD33, and CD38. Monocytes ( 25\%) were positive for CD2, CD4, CD11b, CD11c, CD13, CD14, CD33, CD38, CD45, CD64, and HLA-DR. CD56 was negative.

CCAAT/enhancer-binding protein alpha (CEBPA) mutational analysis was not detected. A fluorescence in situ hybridization (FISH) test (Figure 3) showed no evidence of RARA rearrangement, no evidence of BCR/ABL rearrangement, no evidence of PML/RARA gene rearrangement, and no evidence for RUNX1/RUNX1T1 rearrangement; however, a subset of cells showed an abnormal hybridization pattern, consistent with gain of 8q or trisomy 8 (Figure 3D). There was no evidence of MLL gene locus 11q23 translocation and no evidence of CBFB [inversion (16) or translocation $(16 ; 16)$ ] gene rearrangement. 


\section{Cureus}

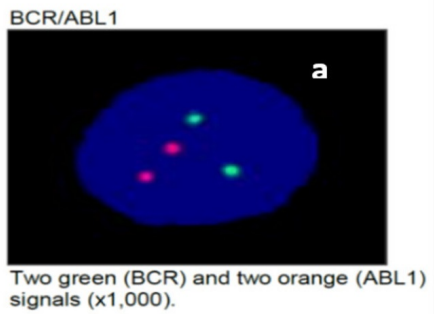

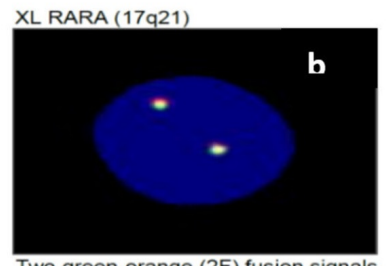

Two green-orange (2F) fusion signals, representing 2 normal RARA loci $(\times 1,000)$.

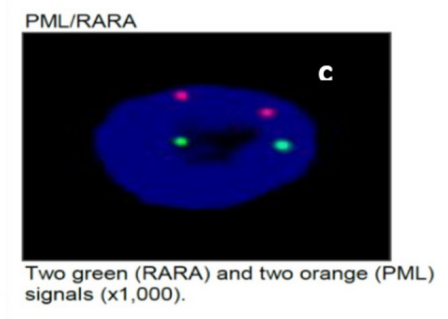

AML1/ETO for $\mathrm{t}(8 ; 21)$

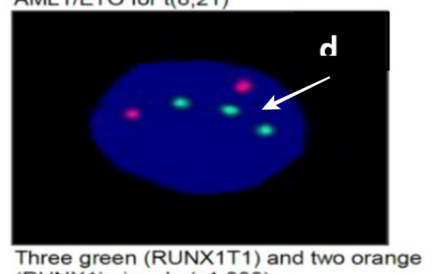

Three green (RUNX1T1)
(RUNX1) signals $(x 1,000)$

\section{FIGURE 3: Fluorescence In Situ Hybridization Results}

Note in (d) three green dots representing three copies of RUNX1T1 and two orange dots representing RUNX1

OnkoSight ${ }^{\mathrm{TM}}$ (Bio-Reference Laboratories, Inc., NJ, USA) NGS AML panel sequencing identified a frameshift mutation in ASXL1 (p.Gly646Trpfs*12), a frameshift mutation in RUNX1 (p.Tyr281Leufs*319), and a hotspot missense mutation in DNMT3A (p.Arg882His). Cytogenetics revealed an abnormal male karyotype with tetrasomy of 8 (49, XY, 8+, 8+) (Figure 4).

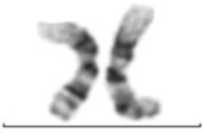

1
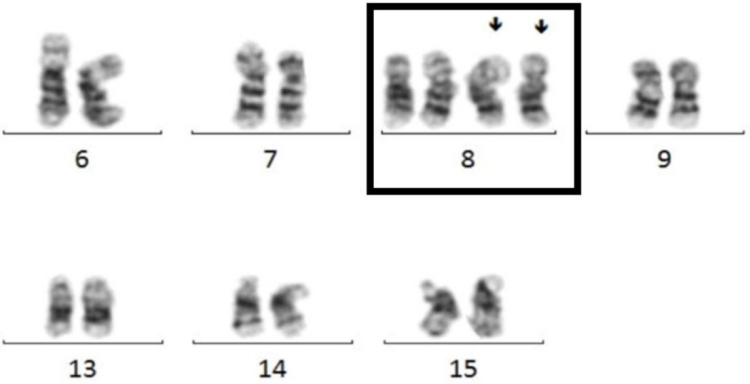

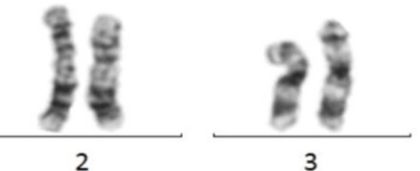

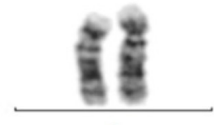

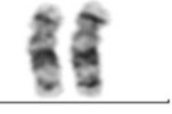

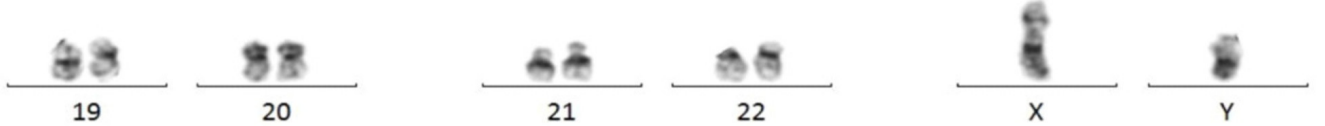

FIGURE 4: Karyotype of the Patient Showing Tetrasomy of

Chromosome 8

\section{Discussion}


Tetrasomy 8 is a rare event in hematologic disorders, associated with monocytic neoplasia ( $50 \%$ of cases). Most frequently, it’s associated with AML-M5 [4]. Other conditions reported in association with isolated tetrasomy 8 include AML-M0, AML-M1, AML- M2, AML M7, MDS, and ALL [5]. Tetrasomy 8, when present, offers a major proliferative advantage and an aggressive course with overall median survival of six to seven months even with intensive therapy [4].

Tsirigotis et al. reported a case of a 25-year-old woman with AML-M5 associated with tetrasomy 8 as the sole chromosomal abnormality [2]. She received induction chemotherapy with cytarabine, idarubicin, and etoposide $(7+3+3)$ after which she achieved complete remission but then relapsed in three months. Yan et al. reported two cases, the first one with AML-M4 with tetrasomy 8 as the sole chromosomal abnormality [4]. He received cytarabine and idarubicin but developed systemic mycosis and died on Day 25. The second patient was diagnosed with AML-M2 with two abnormalities. In the first clone, a Ph chromosome was seen as the sole chromosome abnormality, and in the second clone, tetrasomy 8 was detected in association with additional abnormalities including double Ph chromosomes, trisomy 18 , and disomy Y. He was treated with cytarabine and idarubicin and entered into remission. However, he relapsed in three months. He was treated again but he didn't respond and died nine weeks later of an aspergillus lung infection. Aktas et al. reported another case of a 14-year-old girl with AML-M7 with tetrasomy 8 and trisomy 6, 9 and 12 as secondary changes. She initially achieved remission but relapsed in five months and died of intracranial bleeding [6].

Tetrasomy 8 can occur by either of two mechanisms: (a) two consecutive events of single nondisjunction of chromosome 8 or (b) a single event of double non-disjunction of chromosome 8 [2]. No metaphases with trisomy 8 were observed in our patient. The absence of trisomy 8 might be a result of the longer mitotic duration of tetrasomic cells or of the higher proliferative advantage of tetrasomic cells compared to trisomic cells [7]. It is not clear what genes on chromosome 8 play a role in the pathogenesis of this disease or how it may affect the relapse [8]. However, some genes that might be involved in leukemogenesis on chromosome 8 are the following: MYC in 8q24, MOS in 8q22, and RUNX1T1 [9, 10]. These should be considered as potential causes of malignant transformation but further studies are required to prove this and the mechanisms involved with the malignant transformation [11].

\section{Conclusions}

In this report we shared our findings of an unusual phenomenon and how we reached the diagnosis. What was first perceived as a simple case of sepsis turned out to be one of the rarest aberrancies in myeloid disorders. Tetrasomy 8 is a rare genetic abnormality in hematologic disorders including acute myelogenous leukemia. It is an independent poor prognostic marker in patients with acute myelogenous leukemia. The ongoing improvements in molecular and cytogenetic approaches will provide further information on the exact role of tetrasomy 8 in leukemogenesis.

\section{Additional Information Disclosures}

Human subjects: Consent was obtained by all participants in this study. Conflicts of interest: In compliance with the ICMJE uniform disclosure form, all authors declare the following:

Payment/services info: All authors have declared that no financial support was received from any organization for the submitted work. Financial relationships: All authors have declared that they have no financial relationships at present or within the previous three years with any organizations that might have an interest in the submitted work. Other relationships: All authors have declared that there are no other relationships or activities that could appear to have influenced the submitted work. 


\section{References}

1. Bakshi SR, Brahmbhatt MM, Trivedi PI, et al.: Trisomy 8 in leukemia: a GCRI experience . Indian J Hum Genet. 2012, 18:106-108.

2. Tsirigotis P, Papageorgiou S, Abatzis D, et al.: Acute myelogenous leukemia with tetrasomy 8 is a disease with a poor prognosis. Cancer Genet Cytogenet. 2005, 161:78-81.

10.1016/j.cancergencyto.2004.12.016

3. Trautmann U, Gramatzki M, Krauss M, Friz A, Liehr T, Gebhart E: Tetrasomy 8 as a clonal anomaly in myeloid neoplasias. Cancer Genet. 1994, 72:101-104. 10.1016/01654608(94)90123-6

4. Yan J, Marceau D, Drouin R: Tetrasomy 8 is associated with a major cellular proliferative advantage and a poor prognosis: two cases of myeloid hematologic disorders and review of the literature. Cancer Genet Cytogenet. 2001, 125:14-20. 10.1016/S0165-4608(00)00352-6

5. La Starza R, Crescenzi B, Matteucci C, Martelli MF, Mecucci C: Cytogenetic and FISH investigations on tetrasomy 8 in ANLL. Cancer Genet Cytogenet. 1995, 79:182-185. 10.1016/0165-4608(94)00120-Z

6. Aktas D, Tuncbilek E, Cetin M, Hicsonmez G: Tetrasomy 8 as a primary chromosomal abnormality in a child with acute megakaryoblastic leukemia: a case report and review of the literature. Cancer Genet Cytogenet. 2001, 126:166-168. 10.1016/S0165-4608(00)00403-9

7. de Oliveira FM, Brandao RA, Leite-Cueva SD, de Paula Careta F, Simoes BP, Rego EM, Falcao RP: Tetrasomy 8 in a patient with chronic lymphocytic leukemia . Cancer Genet Cytogenet. 2010, 198:166-169. 10.1016/j.cancergencyto.2009.12.016

8. Qumsiyeh MB, Cheng C, Eyre J, Mann KP, Zhang X-X: Tetrasomy 8 evolving into a segmental triplication 8q in a case of acute monocytic leukemia. Cancer Genet Cytogenet. 2000, 116:7476. 10.1016/S0165-4608(99)00091-6

9. Bruckert P, Kappler R, Scherthan H, Link H, Hagmann F-G, Zankl H: Double minutes and cMYC amplification in acute myelogenous leukemia: are they prognostic factors?. Cancer Genet Cytogenet. 2000, 120:73-79. 10.1016/S0165-4608(99)00235-6

10. Roumier C, Fenaux P, Lafage M, Imbert M, Eclache V, Preudhomme C: New mechanisms of AML1 gene alteration in hematological malignancies. Leukemia. 2003, 17:9-16. 10.1038/sj.leu.2402766

11. Kim J, Park TS, Song J, Lee KA, Lee SG, Cheong JW, Choi JR: Tetrasomy 8 in a patient with acute monoblastic leukemia. Korean J Lab Med. 2008, 28:262-266. 10.3343/kjlm.2008.28.4.262 